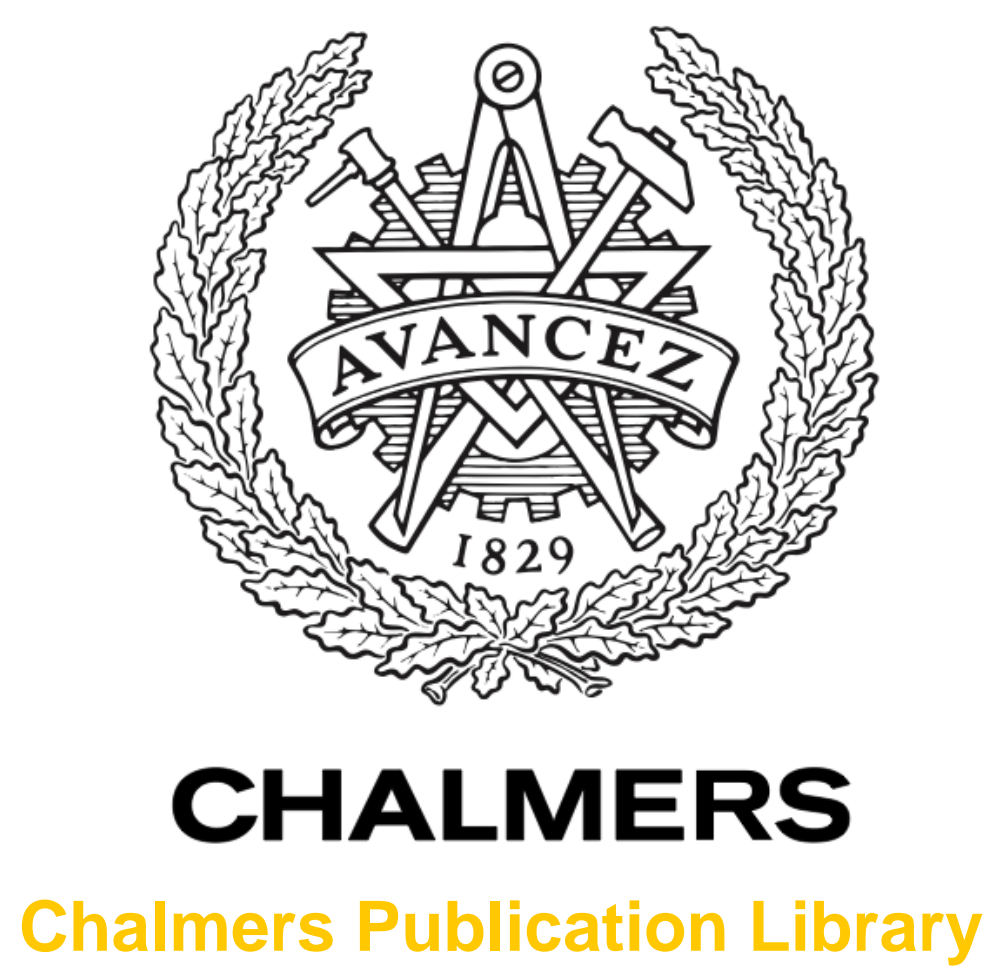

\title{
Characteristic basis function patterns - a novel expansion method for the fast and accurate prediction of antenna array beams
}

This document has been downloaded from Chalmers Publication Library (CPL). It is the author's version of a work that was accepted for publication in:

2012 International Conference on Electromagnetics in Advanced Applications (ICEAA), Cape Town, WP, South Africa, 2-8 Sept 2012

Citation for the published paper:

Maaskant, R. ; Ivashina, M. (2012) "Characteristic basis function patterns - a novel expansion method for the fast and accurate prediction of antenna array beams". 2012 International Conference on Electromagnetics in Advanced Applications (ICEAA), Cape Town, WP, South Africa, 2-8 Sept 2012 pp. 796-799.

http://dx.doi.org/10.1109/ICEAA.2012.6328739

Downloaded from: http://publications.lib.chalmers.se/publication/163393

Notice: Changes introduced as a result of publishing processes such as copy-editing and formatting may not be reflected in this document. For a definitive version of this work, please refer to the published source. Please note that access to the published version might require a subscription. 


\title{
Characteristic Basis Function Patterns - A Novel Expansion Method for the Fast and Accurate Prediction of Antenna Array Beams
}

\author{
R. Maaskant* M. Ivashina ${ }^{\dagger}$
}

\begin{abstract}
A novel beam expansion method is proposed for the fast and accurate prediction of antenna radiation patterns. Only a few physics-based entiredomain vector basis functions - called Characteristic Basis Function Patterns (CBFPs) - are employed for modeling each far-field beam. To determine the beam expansion coefficients, it suffices to measure the unknown beam for a few angular directions only, after which the beam shape is modeled over a large angular region through the interpolatory CBFPs. In practical systems where multiple embedded element- or array-beams are correlated to form a covariace matrix, it even suffices to perform a single measurement on multiple independent reference point sources in the antenna's field of view. This is an important step forward for the fast and accurate calibration of antenna beams, in particular for imaging array systems. An illustrative example is presented on both the procedure for generating the CBFPs and determining their expansion coefficients.
\end{abstract}

\section{INTRODUCTION}

Optimal beam shaping and multi-beaming through the use of advanced antenna array signal processing algorithms are being increasingly applied in various radar, satellite and mobile communication systems, and since recently in radio telescopes and microwave imaging instruments. These antenna arrays offer the flexibility to match the shape of the antenna beam to a required contour, and to maximize the gain or receiving sensitivity of multiple pencil beams with controlled side-lobe and crosspolarization levels.

However, the realized beam pattern may be different in practice from the ideally expected one; for instance, the antenna system may be exposed to temperature variations, mechanical forces, or other temporal perturbations as a result of which the electrical properties of the antenna system may drift. Correction for these drifts requires a complex calibration procedure that is realized in both hardware and software. In practice, it involves several measurements of the gain and/or the pattern shape -

${ }^{*}$ Chalmers University of Technology, Dept. of Signals and Systems, 412 96, Gothenburg , Sweden, e-mail: rob.maaskantechalmers.se

${ }^{\dagger}$ Chalmers University of Technology, Dept. of Signals and Systems, 412 96, Gothenburg, Sweden, e-mail: marianna.ivashina@chalmers.se performed at regular time intervals and spatial directions - and can consume up to $50 \%$ of the total operation time.

In this paper, we propose a novel methodology for the rapid characterization of antenna beam patterns through expanding each (unknown) beam in terms of Characteristic Basis Function Patterns $(\mathrm{CBFPs})^{1}$. The set of CBFPs is generated for a specific EM structure and therefore contains physicsbased information about the antenna system. Furthermore, the CBFPs are chosen to model only specific type of beam errors that are likely to occur, so that only a few CBFPs are required for modeling each beam. Hence, the proposed beam modeling methodology holds the promise to expedient existing beam calibration schemes by 1-2 orders of magnitude and is therefore believed to constitute an important contribution in developing effective calibration methods for large-scale antenna systems and complex beamforming scenarios.

The paper starts by explaining the general concept of generating a set of CBFPs for modeling an unknown beam. Afterwards, the measurement methodology is described to determine the CBFP expansion coefficients using only a few reference sources in the sky. Finally, some illustrative examples are given demonstrating the powerfulness of the novel beam prediction method.

\section{METHODOLOGY - CHARACTERIS- TIC BASIS FUNCTION PATTERNS}

\subsection{CBFP Generation}

We provide a brief description of the method to generate CBFPs and to determine their expansion coefficients. Analogously to the Characteristic Basis Function Method for modeling the current [1], the $m$ th unknown antenna array beam pattern - or embedded element pattern $(\mathrm{EEP})-\boldsymbol{F}^{[m]}(\theta, \phi)$, is expanded into a relatively small set of $N^{[m]}$ CBFPs

\footnotetext{
${ }^{1} \mathrm{~A}$ "beam" can refer to an embedded element pattern or a beamformed array pattern.
} 


$$
\begin{aligned}
\left\{\boldsymbol{G}_{n}^{[m]}(\theta, \phi)\right\}_{n=1}^{N^{[m]}} \text {. Thus, } & \\
\boldsymbol{F}^{[m]}(\theta, \phi) & =\sum_{n=1}^{N^{[m]}} \alpha_{n}^{[m]} \boldsymbol{G}_{n}^{[m]}(\theta, \phi)
\end{aligned}
$$

for $m=1,2, \ldots, M$ beams, where $\left\{\alpha_{n}^{[m]}\right\}$ is the $m$ th set of expansion coefficients, yet to be determined. Although Eq. (1) is a general series expansion, the powerful property of CBFPs is that relatively few CBFPs are required for the accurate modeling of the beams, because:

- CBFPs are physics-based basis function patterns that include a priori information about the antenna system. For instance, for the $m$ th beam pattern, $\boldsymbol{F}^{[m]}$, the primary CBFP, $G_{1}^{[m]}$, is chosen to be as close as possible to the ideally expected beam pattern in the absence of system errors. This CBFP can therefore be determined through physics-based EM simulations or measurements to match the actual expected beam best.

- Higher-order CBFPs, $\boldsymbol{G}_{n}^{[m]}$, for $n>1$, must be chosen to compensate for expected beam errors. Since the primary CBFP is assumed to model the beam in the absence of system errors, higher-order CBFPs are supposed to compensate for beam errors, i.e., they should span the most dominant subspace of expected errors for the $m$ th beam pattern in order to be most effective. Example 1: if the antenna geometry is exposed to mechanical forces, it is recommended to determine the patterns for several deformed geometries and employ these as higher-order CBFPs, because the actual pattern for any geometrical deformation is likely to be a linear superposition of the primary and higher-order CBFPs. Example 2: if an impedance matching error is to be expected, and one aims to predict an EEP in an antenna array, it is natural to employ the EEPs of neighboring antenna elements as higher-order CBFPs to model the perturbed EEP under consideration; since matching errors effectively result in excitations on the neighboring elements causing the EEP under consideration to be modified by a weighted sum of the EEPs of the adjacent antenna elements.

- In a multi-level context, each of the sampled CBFPs can, in turn, be expanded in analytical basis function patterns. Because CBFPs are measured or simulated for discrete directions only, each of the angular sampled CBFPs in (1) can, in turn, be expanded in analytical basis function patterns with fixed expansion coefficients to ease pattern interpolation at any given direction, without increasing the degrees of freedom (DoFs) to solve for.

It is pointed out that, in some cases, scanned array patterns - or EEPs otherwise - may be assumed close to identical in certain array configurations, or be related through simple relations, so that the DoFs in Eq. (1) can be reduced significantly. Example: when the array beam can be modeled by the array factor multiplied by an EEP, only a single EEP needs to be expanded in CBFPs.

Also, it is worthwhile to realize that, in order to minimize the total number of unknown coefficients, the CBFPs should span only the physically relevant subspace of possibly expected patterns. This basis is therefore incomplete in a mathematical sense as it does not model any arbitrary pattern - but is sufficiently complete from a physical point of view.

Finally, note that a set of CBFPs needs to be generated only once, as opposed to the set of expansion coefficients which may need regular updates during operation.

\subsection{Expansion Coefficient Determination}

\subsubsection{Method 1:}

A straightforward manner to determine the $N^{[m]}$ complex-valued expansion coefficients $\left\{\alpha_{n}^{[m]}\right\}_{n=1}^{N^{[m]}}$ in (1) for the $m$ th antenna beam is to sequentially measure the corresponding beam voltage responses for $P$ independent reference point sources in the sky (point matching), where $P \geq N^{[m]}$. Thus, if $\mathrm{V}_{p}^{[m]}$ denotes the open-circuited voltage of the $m$ th beam in the receiving situation for the $p$ th far-field source in the direction $\Omega_{p}$, then

$$
\begin{aligned}
\mathrm{V}_{p}^{[m]} & \propto \boldsymbol{F}^{[m]}\left(\Omega_{p}\right) \cdot \boldsymbol{E}^{\mathrm{i}}\left(\Omega_{p}\right) \\
& =\sum_{n=1}^{N^{[m]}} \alpha_{n}^{[m]}\left[\boldsymbol{G}_{n}^{[m]}\left(\Omega_{p}\right) \cdot \boldsymbol{E}^{\mathrm{i}}\left(\Omega_{p}\right)\right],
\end{aligned}
$$

for $p=1,2, \ldots, P$ directions, and where $\boldsymbol{E}^{\mathrm{i}}\left(\Omega_{p}\right)$ is the complex-valued incident plane wave field from the $p$ th source/direction. The pattern $\boldsymbol{F}^{[m]}$ is defined by exciting the $m$ th beam port by a unit current source, while all other beam ports are open-circuited. From (2), we conclude that the CBFP expansion coefficient vector $\boldsymbol{\alpha}^{[m]}=$ $\left[\alpha_{1}^{[m]}, \alpha_{2}^{[m]}, \ldots, \alpha_{M}^{[m]}\right]^{T}$ can be found by solving the linear system $\mathbf{A}^{[m]} \boldsymbol{\alpha}^{[m]}=\mathbf{V}^{[m]}$, where $A_{p n}^{[m]}=$ $\boldsymbol{G}_{n}^{[m]}\left(\Omega_{p}\right) \cdot \boldsymbol{E}^{\mathrm{i}}\left(\Omega_{p}\right)$. The matrix $\mathbf{A}^{[m]}$ is of size 
$P \times N^{[m]}$, and because $P \geq N^{[m]}$, the solution to the linear system of equations is given through the Moore-Penrose pseudoinverse

$$
\boldsymbol{\alpha}^{[m]}=\left(\left(\mathbf{A}^{[m]}\right)^{H} \mathbf{A}^{[m]}\right)^{-1}\left(\mathbf{A}^{[m]}\right)^{H} \mathbf{V}^{[m]} .
$$

Note that the matrix condition number of $\mathbf{A}^{[m]}$ depends on the position of the sources, which, in turn, may affect the solution accuracy as explained in [2].

\subsubsection{Method 2:}

In "Method 1" we assumed that the distant complex-valued reference point sources are deterministic in both amplitude and phase. In practice, however, the sources may be natural incoherent power point sources that have, statistically seen, no time-averaged phase relation. Then, by using (2), one can instead measure and model the time-averaged beam output power $\left\langle\left|\mathrm{V}_{n}^{[m]}\right|^{2}\right\rangle$. However, since this quantity is real-valued, at least $2 N^{[m]}$ measurements are required to find the $N^{[m]}$ complex-valued CBFP expansion coefficients. Also, solving for voltage beams through power relations requires the solution of a non-linear system of equations.

Instead of observing one point source, one can have multiple incoherent point sources within the antenna's FoV, so that these sources are measured simultaneously. Then, by using an array antenna in conjunction with a complex correlator at the antenna output ports, one can model and measure the $Q \times Q$ antenna output covariance matrix $\mathbf{R}$, where $Q \geq P \geq N^{[m]}$ is the number of antenna array elements, and where element $\mathrm{R}_{p q}=\left\langle\mathrm{V}_{p}^{[m]}\left(\mathrm{V}_{q}^{[m]}\right)^{\star}\right\rangle$ for $\{p, q\} \in\{1,2, \ldots, Q\}$. As detailed in [2], we then fit the modeled antenna output covariance matrix to the measured one to determine the complex-valued expansion coefficients. This again is a non-linear fitting problem.

Finally, and for completeness, we mention that the case of distributed sky reference sources is briefly discussed in [2] as well.

\section{ILLUSTRATIVE EXAMPLE}

Since "Method 2" in the previous section has been largely detailed in [2], we provide an example for the readily comprehensible "Method 1" instead. However, it is by no means limited to this specific example, as already described above.

Fig. 1 illustrates a $3 \times 1$ Vivaldi array with beamforming network for maximizing the gain in a desired direction; in this case $\theta=\phi=0$ (see the ideal beam pattern in Fig. 1). However, the actual
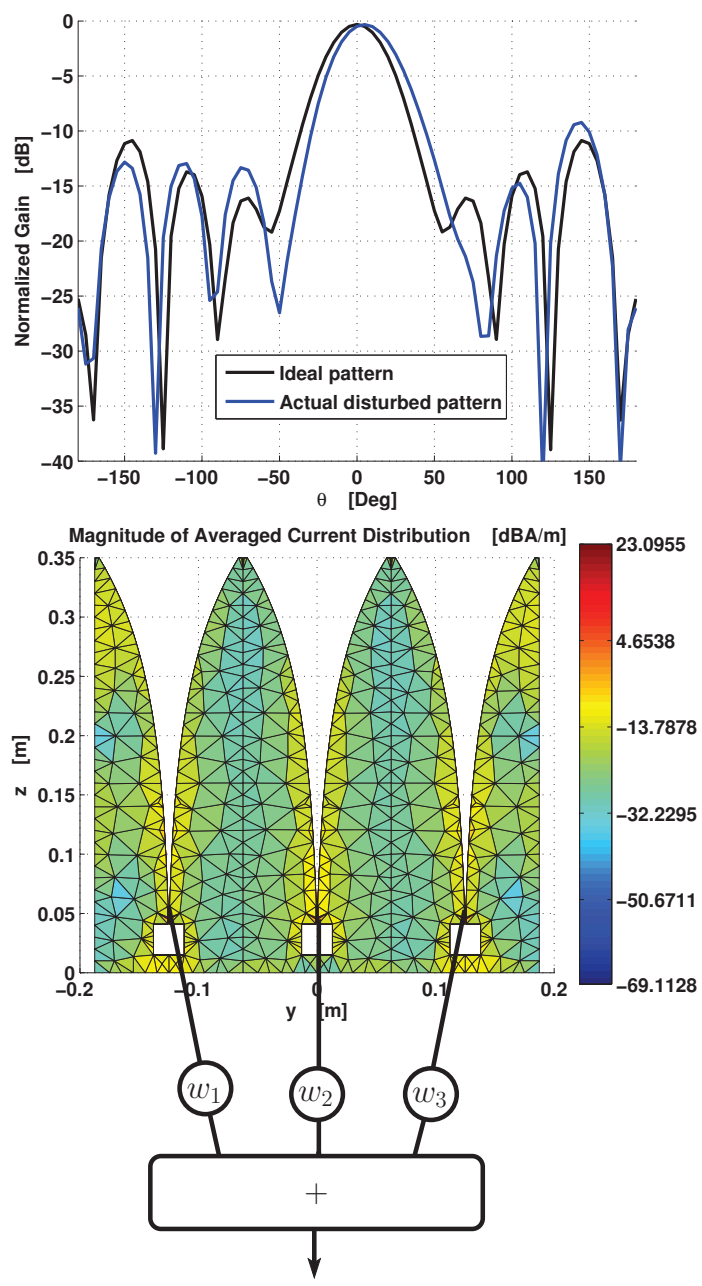

Figure 1: Vivaldi array $(3 \times 1)$ with beamforming network; ideally expected beam pattern, and actual deformed beam.

beamformer weights may drift in practice, e.g. due to temperature variations and/or hardware imperfections. We will therefore assume that the actual beam has a gain maximimum in the $\theta=5^{\circ}$ direction ( $c f$. Fig. 1). The objective is to model the actual beam through only a few CBFPs and calibration sources in the sky.

Following the above proposed procedure for generating the CBFPs, the primary CBFP is chosen to be the ideal broadside-scanned beam as indicated in Fig. 2 (black curve), since this pattern is assumed to be close to the actual beam pattern (blue curve). The higher-order CBFPs are supposed to minimize the beam error. Since the error is expected to be in the beamformer weights, we generate CBFPs through simulations by adjusting the beamformer weights and scan to two offset directions, for example to $\theta_{0}=-10^{\circ}$ and $\theta_{0}=+10^{\circ}$. The corresponding two additional pat- 


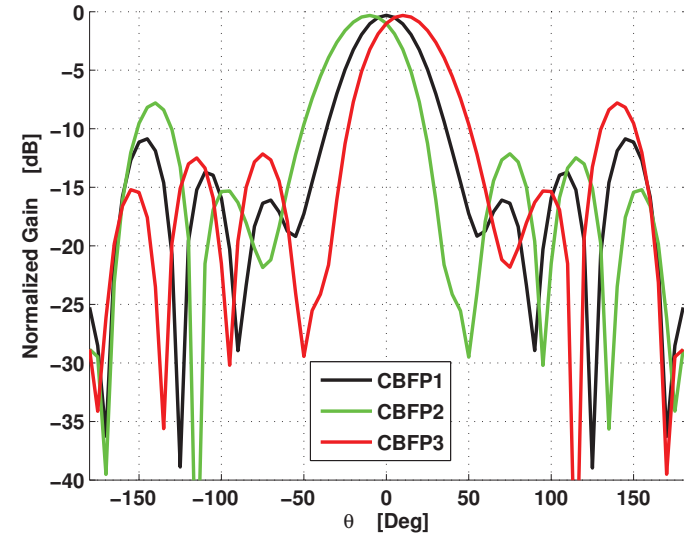

Figure 2: Three CBFPs are generated.

terns are then employed together with the primary one to form the set of 3 CBFPs as shown in Fig. 2. The next step is to determine the 3 expansion coefficients for these CBFPs to predict the actual beam. Toward this end, we measure the actual pattern using 3 reference point sources, for example for $\theta \in\left\{-10^{\circ}, 0^{\circ},+10^{\circ}\right\}$. The actual subsequently measured beam voltages form the $3 \times 1$ right-handside vector $\mathbf{V}^{[1]}$. The $3 \times 3$ matrix $\mathbf{A}^{[1]}$ is constructed by sampling each of the 3 CBPFs in the 3 source directions. Accordingly the CBFP expansion coefficient vector is computed, $\boldsymbol{\alpha}^{[1]}=\left(\mathbf{A}^{[1]}\right)^{-1} \mathbf{V}^{[1]}$, so that the predicted beam can be determined.

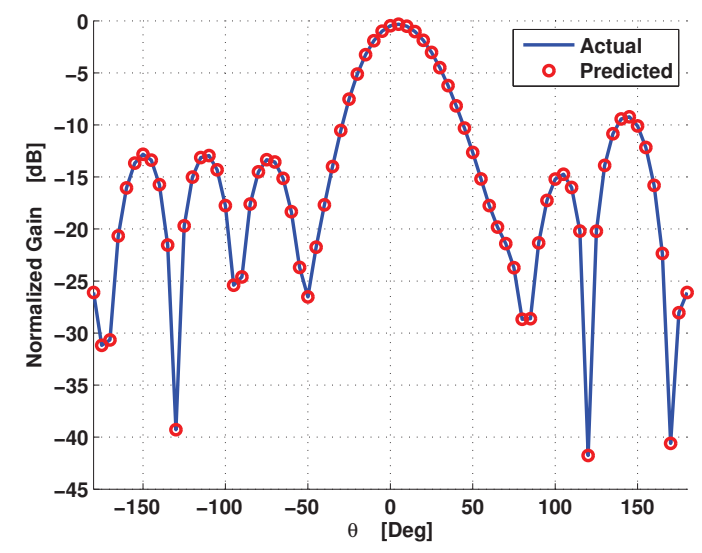

Figure 3: Actual and predicted beam patterns.

Fig. 3 shows that the actual and the predicted beam patterns are identical. This is to be expected, since the pattern for any weight vector can be constructed from a linear combination of the 3 embedded element patterns - or in this case 3 independent array-scanned beams.
Many other examples and scenerios can be analyzed with the proposed beam modeling concept. One example: if an error in the element geometry is to be expected, CBFPs can be generated for several deformed geometries and then be used to predict the actual beam. However, one should realize that the relation between geometrical variations and the beam shape is a non-linear one, as opposed to variations of the beamformer weights on the beam pattern. If the errors are small, however, one can linearize the problem so that the proposed modeling method is still valid, albeit in approximated form.

\section{CONCLUSIONS}

A novel expansion method is proposed that requires to employ only a small number of physics-based characteristic basis function patterns (CBFPs) for modeling antenna beams. Only few CBFPs are required since they incorporate the electromagnetic radiation properties of the antenna system and exploit a priori knowledge on the type of beam errors that are to be expected in practice. The CBFP expansion coefficients are determined by measuring on only a few far-field reference sources in the sky. An illustrative example is shown which demonstrates the methodology in one of its simplest forms.

\section{Acknowledgments}

We would like to express our gratitude to our collaborators on this topic: Dr. Stefan Wijnholds, Prof. Karl Warnick, Oleg Iupikov, Andre Young, and Prof. David Davidson. This work is is carried out within the research programme Rubicon, which is partly financed by the Netherlands Organization for Scientific Research (NWO), and VINNOVA, which is the Swedish Agency for Innovation Systems.

\section{References}

[1] J. Yeo, V. Prakash, and R. Mittra, "Efficient analysis of a class of microstrip antennas using the characteristic basis function method (CBFM)," Micr. Opt. Technol., vol. 39, pp. 456-464, Dec. 2003.

[2] R. Maaskant, M. V. Ivashina, S. J. Wijnholds, and K. F. Warnick, "Efficient prediction of array element patterns using physics-based expansions and a single far-field measurement," IEEE Trans. Antennas Propag., accepted for publication, 2012. 